PROCEEDINGS OF THE AMERICAN MATHEMATICAL SOCIETY

Volume 127, Number 4, April 1999, Pages 1151-1161

S 0002-9939(99)04992-8

\title{
FINITE RANK PERTURBATIONS AND DISTRIBUTION THEORY
}

\author{
S. ALBEVERIO AND P. KURASOV
}

(Communicated by David R. Larson)

\begin{abstract}
Perturbations $A_{T}$ of a selfadjoint operator $A$ by symmetric finite rank operators $T$ from $\mathcal{H}_{2}(A)$ to $\mathcal{H}_{-2}(A)$ are studied. The finite dimensional family of selfadjoint extensions determined by $A_{T}$ is given explicitly.
\end{abstract}

\section{INTRODUCTION}

Finite rank perturbations of selfadjoint operators are used in quantum mechanics to construct exactly solvable models for different physical problems (see [2]). The resolvents of any selfadjoint operator $A$ and its finite rank perturbation $A_{T}=A+T$ are related by M.Krein's formula [17, 1], since these two selfadjoint operators are extensions of one symmetric operator. The symmetric operator coincides with the restriction of the original operator $A$ to the set of elements from the kernel of the finite dimensional operator $T$. If the finite dimensional operator $T$ is a bounded operator in the Hilbert space, then the domains of the perturbed and original operators coincide. We are going to concentrate our attention on the finite rank perturbations determined by the operator $T$ acting in the rigged Hilbert spaces $\mathcal{H}_{2}(A) \rightarrow \mathcal{H}_{-2}(A)$ associated with the operator $A$. The perturbation in the latter case can be defined using the method of quadratic forms. Thus if the quadratic form associated with the operator $T$ is infinitesimally bounded with respect to the quadratic form of the operator $A$, then the operator $A_{T}$ can be determined using the KLMN theorem ([18]). If the operator $T$ does not have the property described above, then the perturbed operator is not defined uniquely. It is not clear which selfadjoint extension of the restricted operator corresponds to the formal sum $A+T$. Therefore the selfadjoint perturbed operator is determined by a set of additional parameters.

The corresponding problem for rank one perturbations

$$
A_{\alpha}=A+\alpha\langle\varphi, \cdot\rangle \varphi, \quad \varphi \in \mathcal{H}_{-2}(A), \alpha \in \mathbf{R},
$$

has been intensively studied during the last few years $[3,4,5,6,9,10,11,13,14$, $16,19]$. It has been shown in $[5,6]$ that the set of additional parameters in this case consists of one parameter only. The unique additional parameter in fact determines the choice of the $Q$-function appearing in M.Krein's formula. Krein's $Q$-function is

Received by the editors August 1, 1997.

1991 Mathematics Subject Classification. Primary 34L40, 46F10, 47A55, 81Q15.

(C)1999 American Mathematical Society 
determined by the relation

$$
\frac{Q(\lambda)-Q(\mu)}{\lambda-\mu}=\left\langle\frac{1}{A-\lambda} \varphi, \frac{1}{A-\mu} \varphi\right\rangle
$$

and therefore contains one arbitrary additive parameter. It determines the parameterization of the one dimensional family of the selfadjoint extensions. The problem described above can be understood as the regularization problem for the quadratic form of the resolvent. Consider the scale of Hilbert spaces $\mathcal{H}_{n}(|A|)$ associated with the positive operator $|A|$. Let $\varphi \in \mathcal{H}_{-1}(|A|)$; then Krein's $Q$-function can be chosen to be equal to

$$
Q(\lambda)=\left\langle\varphi, \frac{1}{A-\lambda} \varphi\right\rangle
$$

The latter equality determines a natural choice of the arbitrary additive parameter. If $\varphi \in \mathcal{H}_{-2}(A) \backslash \mathcal{H}_{-1}(|A|)$, then the scalar product $\left\langle\varphi, \frac{1}{A-\lambda} \varphi\right\rangle$ is not always defined. The latter quadratic form can formally be written as follows:

$$
\left\langle\varphi, \frac{1}{A-\lambda} \varphi\right\rangle=\left\langle\varphi, \frac{A}{A^{2}+1} \varphi\right\rangle+\left\langle\varphi, \frac{1+\lambda A}{\left(A^{2}+1\right)(A-\lambda)} \varphi\right\rangle .
$$

The second scalar product on the right hand side of the latter formula is well defined. Therefore to define the scalar product which appears on the left hand side it is enough to determine the real parameter $\left\langle\varphi, \frac{A}{A^{2}+1} \varphi\right\rangle$. This parameter is uniquely defined in the case $\left.\varphi \in \mathcal{H}_{-1}(|A|)\right)$ and can in general be chosen arbitrary in the case $\varphi \in \mathcal{H}_{-2}(A) \backslash \mathcal{H}_{-1}(|A|)$. It has been proven in $[5,6]$ that the parameter can be determined uniquely even in the latter case if the original operator $A$ and the vector $\varphi$ are homogeneous with respect to a certain one-parameter group of unitary transformations of the Hilbert space. Then there exists a unique homogeneous extension of the functional $\varphi$ to the element $\frac{A}{A^{2}+1} \varphi$. These methods permit us to construct approximations of unbounded perturbations by bounded ones.

In the present paper we extend the methods described above to include finite rank perturbations. More precisely, we show that every symmetric finite rank perturbation of a selfadjoint operator $A$ is determined by a finite set of vectors $\varphi_{n} \in \mathcal{H}_{-2}(A), n=1,2, \ldots, N$, and a Hermitian $N \times N$ matrix. The $Q$-matrix appearing in Krein's formula contains an arbitrary $N \times N$ Hermitian matrix R. It is proven that the perturbed operator is uniquely defined if all $\varphi_{n}$ are elements from $\mathcal{H}_{-1}(|A|)$, i.e., there exists a natural choice of the matrix $\mathrm{R}$ in this case. If the latter condition is not satisfied then the perturbed operator is determined by a certain $N \times N$ Hermitian matrix R. We discuss the question of when the elements of the latter matrix cannot be chosen arbitrary. The case of homogeneous vectors $\varphi_{n}$ is also studied.

In section 2 we give the definition of perturbations $A_{T}$ of selfadjoint operators $A$ by finite rank operators $T$ from $\mathcal{H}_{2}(A)$ to $\mathcal{H}_{-2}(A)$ and give the general form of the associated selfadjoint extensions. In section 3 we consider in detail the case where $T$ is form bounded with respect to $A$. In section 4 we consider in detail the case where $T$ is not form bounded with respect to $A$. We show that a certain Hermitian matrix satisfying a certain admissibility condition determines the perturbation. 


\section{DEFINITION AND GENERAL CONSTRUCTION OF THE PERTURBED OPERATORS}

Additive finite rank perturbations of a selfadjoint operator $A$ acting in the (complex) Hilbert space $\mathcal{H}$ are given formally by

$$
A_{T}=A+T
$$

where the operator $T$ is a finite dimensional operator acting from the Hilbert space $\mathcal{H}_{2}(A)$ to the Hilbert space $\mathcal{H}_{-2}(A)$. We consider the case where $T$ is a symmetric operator acting in those Hilbert spaces, i.e. for every two functions $u, v \in \mathcal{H}_{2}(A)$ the following equality holds:

$$
\langle u, T v\rangle=\langle T u, v\rangle
$$

The fact that the image of the operator $T$ has finite dimension implies that there exist linearly independent elements $\varphi_{j}, j=1,2, \ldots, M$, from the space $\mathcal{H}_{-2}(A)$ which span the image space, i.e., the following formula holds:

$$
T u=\sum_{j=1}^{M} \psi_{j}(u) \varphi_{j}
$$

where $\psi_{j}$ are certain linear bounded functionals on $\mathcal{H}_{2}(A)$. Formula (2) implies that the functionals $\psi_{j}$ are equal to linear combinations of the functionals $\varphi_{j}$, i.e. there exists a matrix $\mathbf{T}=\left\{t_{i j}\right\}_{i, j=1}^{M}, t_{i j} \in \mathbf{C}$, such that

$$
\psi_{j}=\sum_{i=1}^{M} \overline{t_{j i}} \varphi_{i}
$$

Moreover, the latter representation and formula (2) imply that

$$
0=\langle u, T v\rangle-\langle T u, v\rangle=\sum_{j, i=1}^{M}\left(t_{j i}-\overline{t_{i j}}\right)\left\langle\varphi_{j}, v\right\rangle \overline{\left\langle\varphi_{j}, u\right\rangle}
$$

Therefore the matrix $\mathbf{T}$ must in fact be Hermitian, $t_{j i}=\overline{t_{i j}}$. Thus every symmetric finite rank perturbation of a selfadjoint operator is determined by a finite set of elements $\varphi_{j}, j=1,2, \ldots, M$, from the Hilbert space $\mathcal{H}_{-2}(A)$ and a Hermitian $M \times M$ matrix $\mathbf{T}=\left\{t_{i j}\right\}_{i, j=1}^{M}$. We suppose in addition that the matrix $\mathbf{T}$ is invertible. The latter assumption does not restrict the set of perturbations considered. If the matrix $\mathbf{T}$ is not invertible, i.e., has zero determinant, then let us denote by $N$ the kernel of $\mathbf{T}$. Considering the orthogonal complement to the subspace $N$, we get a finite rank operator of order less than $M$ determined by a nondegenerate Hermitian matrix. Thus every additive symmetric finite rank perturbation of the operator $A$ is given on the domain of $A$ by

$$
A_{T}=A+\sum_{i, j=1}^{M} t_{j i}\left\langle\varphi_{i}, \cdot\right\rangle \varphi_{j}
$$

where the matrix $\mathbf{T}$ is invertible and Hermitian and the vectors $\varphi_{j}, j=1, \ldots, M$, are elements from the Hilbert space $\mathcal{H}_{-2}(A)$. We suppose that the vectors $\varphi_{j}$ form an orthonormal system in the Hilbert space $\mathcal{H}_{-2}(A)$ :

$$
\left\langle\frac{1}{A-i} \varphi_{i}, \frac{1}{A-i} \varphi_{j}\right\rangle=\delta_{i j}
$$


where $\delta_{i j}$ is the Kronecker symbol. We suppose in addition that the vectors $\varphi_{j}$ are $\mathcal{H}$-independent, i.e. no nontrivial linear combination of these vectors belongs to the original Hilbert space $\mathcal{H}$.

The selfadjoint operator corresponding to the symmetric operator (5) defined on $\operatorname{Dom}(A)$ coincides with one of the selfadjoint extensions of the operator $A$ restricted to the following domain:

$$
\operatorname{Dom}_{M}=\operatorname{Dom}(A) \cap \operatorname{Ker}(T),
$$

where $\operatorname{Ker}(T)$ denotes the kernel of the operator $T$.

It has been proven in $[5,6]$ that the restricted operator is densely defined and has deficiency indices $(1,1)$ in the case $M=1$ (Lemma 2.1 from [6]).

Lemma 1. Suppose that the vectors $\varphi_{j} \in \mathcal{H}_{-2}(A) \backslash \mathcal{H}, j=1,2, \ldots, M$, are $\mathcal{H}$ independent and form an orthonormal system in $\mathcal{H}_{-2}(A)$, i.e. $\left\langle\frac{1}{A-i} \varphi_{j}, \frac{1}{A-i} \varphi_{k}\right\rangle=$ $\delta_{j k}$. Then the restriction $A_{M}^{0}$ of the operator $A$ to the domain $\operatorname{Dom}\left(A_{M}^{0}\right)=\{\psi \in$ $\left.\operatorname{Dom}(A):\left\langle\varphi_{j}, \psi\right\rangle=0, j=1,2, \ldots, M\right\}$ is a densely defined symmetric operator with the deficiency indices $(M, M)$.

Proof. The restricted operator $A_{M}^{0}$ is densely defined, since the vectors $\varphi_{j}$ are $\mathcal{H}$ independent. One can easily prove that the deficiency subspace for $\lambda=i$ coincides with the linear hull of the vectors $\frac{1}{A-i} \varphi_{j}$. Since these vectors are orthogonal, the dimension of the deficiency subspace coincides with the number of basis vectors. It follows that the operator $A_{M}^{0}$ is densely defined and has deficiency indices $(M, M)$. This completes the proof of the lemma.

In the proof of the lemma we have not used the fact that the vectors $\varphi_{j}$ have unit norm in $\mathcal{H}_{-2}(A)$. It was enough to suppose that the vectors are $\mathcal{H}$-independent. But every such system can easily be orthonormalized and has basis elements not belonging to the Hilbert space $\mathcal{H}$. Therefore we are going to use only orthonormal systems $\left\{\varphi_{j}\right\}_{j=1}^{M}$ in what follows.

The condition that the vectors $\varphi_{j}$ are $\mathcal{H}$-independent is not essential. If the set of vectors is not $\mathcal{H}$-independent then the operator $A_{M}^{0}$ is not densely defined. But its selfadjoint extensions can be described by similar formulas.

Every element $\psi$ from the domain of the adjoint operator $\operatorname{Dom}\left(A_{M}^{0 *}\right)$ can be presented in the following form:

$$
\psi=\hat{\psi}+\sum_{j=1}^{M}\left(a_{+j}(\psi) \frac{1}{A-i} \varphi_{j}+a_{-j}(\psi) \frac{1}{A+i} \varphi_{j}\right),
$$

where $\hat{\psi} \in \operatorname{Dom}\left(A_{M}^{0}\right), a_{ \pm j}(\psi) \in \mathbf{C}$. We are going to use the following vector notation:

$$
\vec{a}_{ \pm} \equiv\left\{a_{ \pm j}\right\}_{j=1}^{M}
$$

The adjoint operator $A_{M}^{0 *}$ acts as follows on every $\psi \in \operatorname{Dom}\left(A_{M}^{0 *}\right)$ :

$$
\begin{aligned}
& A_{M}^{0 *}\left(\hat{\psi}+\sum_{j}\left(a_{+j}(\psi) \frac{1}{A-i} \varphi_{j}+a_{-j}(\psi) \frac{1}{A+i} \varphi_{j}\right)\right) \\
& \quad=A \hat{\psi}+\sum_{j=1}^{M}\left(a_{+j}(\psi) \frac{i}{A-i} \varphi_{j}+a_{-j}(\psi) \frac{-i}{A+i} \varphi_{j}\right) .
\end{aligned}
$$


The boundary form of the adjoint operator is given by

$$
\begin{aligned}
& \left\langle A_{M}^{0 *} \psi, \eta\right\rangle-\left\langle\psi, A_{M}^{0 *} \eta\right\rangle \\
& \quad=2 i \sum_{j, k=1}^{M}\left(\overline{a_{-j}(\psi)} a_{-k}(\eta)-\overline{a_{+j}(\psi)} a_{+k}(\eta)\right)\left\langle\frac{1}{A-i} \varphi_{j}, \frac{1}{A-i} \varphi_{k}\right\rangle \\
& \quad=2 i \sum_{j=1}^{M}\left(\overline{a_{-j}(\psi)} a_{-j}(\eta)-\overline{a_{+j}(\psi)} a_{+j}(\eta)\right) \\
& =2 i\left(\left\langle\vec{a}_{-}(\psi), \vec{a}_{-}(\eta)\right\rangle_{\mathbf{C}^{M}}-\left\langle\vec{a}_{+}(\psi), \vec{a}_{+}(\eta)\right\rangle_{\mathbf{C}^{M}}\right),
\end{aligned}
$$

since the vectors $\frac{1}{A-i} \varphi_{j}$ form an orthonormal system.

The selfadjoint extensions of the operator $A_{M}^{0 *}$ can be parameterized by $M \times M$ unitary matrices using the von Neumann theory. Let $V=\left\{v_{j k}\right\}_{j, k=1}^{M}$ be such a matrix. The corresponding selfadjoint operator $A_{V}$ coincides with the restriction of the operator $A_{M}^{0 *}$ to the domain $\operatorname{Dom}_{V}=\left\{\psi \in \operatorname{Dom}\left(A_{M}^{0 *}\right): \vec{a}_{-}(\psi)=V \vec{a}_{+}(\psi)\right\}$. The extension corresponding to the matrix $V=-I$ coincides with the original operator $A$.

To define the finite rank perturbations of $A$ we consider again two scales of Hilbert spaces. The first set of spaces is the standard scale of Hilbert spaces associated with the operator $|A|$ :

$$
\mathcal{H}_{2}(A) \subset \mathcal{H}_{1}(|A|) \subset \mathcal{H} \subset \mathcal{H}_{-1}(|A|) \subset \mathcal{H}_{-2}(A) .
$$

The second scale of Hilbert spaces

$$
\mathcal{H}_{2}(A)=\operatorname{Dom}(A) \subset \mathrm{B}_{M}(A) \subset \mathcal{H} \subset \mathrm{B}_{M}(A)^{*} \subset \operatorname{Dom}(A)^{*}=\mathcal{H}_{-2}(A)
$$

is constructed using the operators $A$ and $T$. Here $\mathrm{B}_{M}(A)$ denotes the domain of the adjoint operator $A_{M}^{0 *}$. The norms in the spaces $\mathcal{H}_{2}(A)$ and $\mathcal{H}_{-2}(A)$ are equal to the standard norms in these Hilbert spaces. The norm in the space $\mathrm{B}_{M}(A)$ is defined using the orthonormal basis in the deficiency subspace. Let $\psi$ be an element from the space $\mathrm{B}_{M}(A)=\operatorname{Dom}\left(A_{M}^{0 *}\right)$; then $\psi$ possesses the representation (6). This representation can be written as follows:

$$
\begin{aligned}
\psi & =\hat{\psi}+\sum_{j=1}^{M}\left(a_{+j}(\psi)\left(\frac{i}{A^{2}+1}+\frac{A}{A^{2}+1}\right) \varphi_{j}+a_{-j}(\psi)\left(\frac{-i}{A^{2}+1}+\frac{A}{A^{2}+1}\right) \varphi_{j}\right) \\
& =\tilde{\psi}+\sum_{j=1}^{M} b_{j}(\psi) \frac{A}{A^{2}+1} \varphi_{j}
\end{aligned}
$$

where

$$
\begin{aligned}
\tilde{\psi} & =\hat{\psi}+\sum_{j=1}^{M}\left(a_{+j}(\psi)-a_{-j}(\psi)\right) \frac{i}{A^{2}+1} \varphi_{j} ; \\
b_{j}(\psi) & =a_{+j}(\psi)+a_{-j}(\psi) .
\end{aligned}
$$

The norm in the space $\mathrm{B}_{M}(A)$ is then defined by the formula

$$
\|\psi\|_{\mathrm{B}_{M}(A)}=\|\tilde{\psi}\|_{\operatorname{Dom}(A)}+\sqrt{\sum_{j=1}^{M}\left|b_{j}(\psi)\right|^{2}} .
$$


The space $\mathrm{B}_{M}(A)$ can be considered as a finite dimensional extension of the space $\operatorname{Dom}(A)$ in the sense that $\mathrm{B}_{M}(A)$ is isomorphic with $\operatorname{Dom}(A) \dot{+} \mathbf{C}^{M}$. The natural norm-preserving embedding $\rho_{M}$ of $\operatorname{Dom}(A) \dot{+} \mathbf{C}^{M}$ into $\mathrm{B}_{m}(A)$ is defined by (9). The norms in the dual spaces are defined correspondingly.

\section{FORM BOUNDED FINITE RANK PERTURBATIONS}

Let the operator $T$ be form bounded with respect to the operator $A$, i.e. the following inequality holds:

$$
\langle\psi, T \psi\rangle \leq a\langle\psi,|A| \psi\rangle+b\langle\psi, \psi\rangle
$$

for every $\psi$ from the domain $\operatorname{Dom}(A)$ and some $a, b \geq 0$. This assumption holds if and only if all the vectors $\varphi_{j}, j=1,2, \ldots, M$, are from the Hilbert space $\mathcal{H}_{-1}(|A|)$. In this section we only consider this case. Let $\varphi_{j} \in \mathcal{H}_{-1}(|A|)$; then

$$
\begin{gathered}
|\langle\psi, T \psi\rangle|=\left|\sum_{i, j=1}^{M} t_{j i}\left\langle\psi, \varphi_{j}\right\rangle\left\langle\varphi_{i}, \psi\right\rangle\right| \\
\leq\left(\sum_{i, j=1}^{M}\left|t_{i j}\right|\left\|\frac{1}{\sqrt{|A|}+i} \varphi_{j}\right\|\left\|\frac{1}{\sqrt{|A|}+i} \varphi_{i}\right\|\right)\|(\sqrt{|A|}-i) \psi\|^{2} \\
=\left(\sum_{i, j=1}^{M}\left|t_{i j}\right|\left\|\varphi_{j}\right\|_{\mathcal{H}_{-1}(|A|)}\left\|\varphi_{i}\right\|_{\mathcal{H}_{-1}(|A|)}\right)(\langle\psi,|A| \psi\rangle+\langle\psi, \psi\rangle),
\end{gathered}
$$

since the norms of the vectors $\frac{1}{\sqrt{|A|}+i} \varphi_{j}$ can be calculated as follows:

$$
\left\|\frac{1}{\sqrt{|A|}+i} \varphi_{j}\right\|^{2}=\left\langle\varphi_{j}, \frac{1}{|A|+1} \varphi_{j}\right\rangle=\left\|\varphi_{j}\right\|_{\mathcal{H}_{-1}(|A|)} .
$$

Theorem 1. Let $\varphi_{j} \in \mathcal{H}_{-1}(|A|) \backslash \mathcal{H}$ form an orthonormal basis in $\mathcal{H}_{-2}(A)$, i.e. $\left\langle\frac{1}{A-i} \varphi_{j}, \frac{1}{A-i} \varphi_{k}\right\rangle=\delta_{j k}$, and let $\mathbf{T}=\left\{t_{i j}\right\}_{i, j=1}^{M}$ be a Hermitian invertible matrix. Then the selfadjoint operator $A_{T}=A+\sum_{i, j=1}^{M} t_{i j}\left\langle\varphi_{j}, \cdot\right\rangle \varphi_{i}$ is the selfadjoint extension of the operator $A_{M}^{0}$ to the domain

$$
\operatorname{Dom}\left(A_{T}\right)=\left\{\psi \in \operatorname{Dom}\left(A_{M}^{0 *}\right): \vec{a}_{+}(\psi)=-\left(\mathbf{T}^{-1}+\Phi\right)^{-1}\left(\mathbf{T}^{-1}+\Phi^{*}\right) \vec{a}_{-}(\psi)\right\},
$$

where $\Phi$ is the $M \times M$ matrix $\Phi_{i j}=\left\langle\varphi_{i}, \frac{1}{A-i} \varphi_{j}\right\rangle, i, j=1,2, \ldots, M$.

Comment. The matrix $\mathbf{T}$ is supposed to be invertible. For $\mathbf{T}=0$ we therefore have $A_{0}=A$.

Proof. The operator $A_{T}$ is defined as a linear operator acting in the Hilbert spaces $\mathrm{B}_{M}(A)=\operatorname{Dom}\left(A_{M}^{0 *}\right) \rightarrow \operatorname{Dom}(A)^{*}$. Let $\psi \in \mathrm{B}_{M}(A)$; then the operator $A_{M}$ acts as 
follows:

$$
\begin{aligned}
A_{M} \psi= & \left(A+\sum_{i, j=1}^{M} t_{i j}\left\langle\varphi_{j}, \cdot\right\rangle \varphi_{i}\right) \\
& \times\left(\hat{\psi}+\sum_{k=1}^{M}\left(a_{+k}(\psi) \frac{1}{A-i} \varphi_{k}+a_{-k}(\psi) \frac{1}{A+i} \varphi_{k}\right)\right) \\
= & A \hat{\psi}+\sum_{i, j=1}^{M} t_{i j}\left\langle\varphi_{j}, \hat{\psi}\right\rangle \varphi_{i} \\
& +\sum_{k=1}^{M}\left(a_{+k}(\psi)\left(\varphi_{k}+\frac{i}{A-i} \varphi_{k}\right)+a_{-k}(\psi)\left(\varphi_{k}-\frac{i}{A+i} \varphi_{k}\right)\right) \\
& +\sum_{i, j, k=1}^{M}\left(a_{+k} t_{i j}\left\langle\varphi_{j}, \frac{1}{A-i} \varphi_{k}\right\rangle \varphi_{i}+a_{-k} t_{i j}\left\langle\varphi_{j}, \frac{1}{A+i} \varphi_{k}\right\rangle \varphi_{i}\right) \\
= & A_{M}^{0 *} \psi+\sum_{k=1}^{M}\left\{a_{+k}+a_{-k}+\sum_{i, j=1}^{M}\left(t_{k j}\left\langle\varphi_{j}, \frac{1}{A-i} \varphi_{i}\right\rangle a_{+i}\right.\right. \\
& \left.\left.+t_{k j}\left\langle\varphi_{j}, \frac{1}{A+i} \varphi_{i}\right\rangle a_{-i}\right)\right\} \varphi_{k} .
\end{aligned}
$$

In the calculations we used the fact that $\left\langle\varphi_{j}, \hat{\psi}\right\rangle=0$. The domain $\operatorname{Dom}\left(A_{T}\right)$ of the selfadjoint operator $A_{T}$ coincides with the following set: $\left\{\psi \in \operatorname{Dom}\left(A_{M}^{0 *}\right)\right.$ : $\left.A_{T} \psi \in \mathcal{H}\right\}$. The element $A_{T} \psi$ belongs to the Hilbert space $\mathcal{H}$ if and only if the expression in the curly brackets is equal to zero, i.e. the following equation is satisfied:

$$
a_{+k}+a_{-k}+\sum_{i, j=1}^{M}\left(t_{k j}\left\langle\varphi_{j}, \frac{1}{A-i} \varphi_{i}\right\rangle a_{+i}+t_{k j}\left\langle\varphi_{j}, \frac{1}{A+i} \varphi_{i}\right\rangle a_{-i}\right)=0 .
$$

The latter equation can be written in matrix form using the notation (7) as follows:

$$
(I+\mathbf{T} \Phi) \vec{a}_{+}=-\left(I+\mathbf{T} \Phi^{*}\right) \vec{a}_{-},
$$

where $I$ denotes the unit $M \times M$ matrix. The matrix $I+\mathbf{T} \Phi$ is invertible, since $\mathbf{T}$ is an invertible Hermitian matrix and the imaginary part of the matrix $\Phi$ is equal to the unit matrix. The latter statement follows from the following formula:

$$
\Phi_{i j}=\Re \Phi_{i j}+i \Im \Phi_{i j}, \quad \Re \Phi_{i j}=\left\langle\varphi_{i}, \frac{A}{A^{2}+1} \varphi_{j}\right\rangle, \Im \Phi_{i j}=I,
$$

which is valid, since the vectors $\varphi_{j}$ form an orthonormal system in the space $\mathcal{H}_{-2}(A)$. Thus the condition (14) can be written in the form

$$
\vec{a}_{+}(\psi)=-\left(\mathbf{T}^{-1}+\Phi\right)^{-1}\left(\mathbf{T}^{-1}+\Phi^{*}\right) \vec{a}_{-}(\psi) .
$$

The matrix $-\left(\mathbf{T}^{-1}+\Phi\right)^{-1}\left(\mathbf{T}^{-1}+\Phi^{*}\right)$ is unitary, and the adjoint operator restricted to the domain of functions satisfying the conditions (16) is selfadjoint.

If the matrix $\mathbf{T}$ is equal to zero, then (13) implies that $\vec{a}_{-}=-\vec{a}_{+}$, and the extended operator is just the original operator $A$. This completes the proof of the theorem.

We consider now the set of all selfadjoint extensions of the operator $A_{M}^{0}$. Every such operator coincides with the restriction of the adjoint operator $A_{M}^{0 *}$ to the domain of functions satisfying boundary conditions of the form

$$
\vec{a}_{+}(\psi)=V \vec{a}_{-}(\psi)
$$


where $V$ is a certain unitary $M \times M$ matrix. The domain of the extended selfadjoint operator coincides with the domain of a certain operator $A_{T}$ if and only if the following equality holds:

$$
V=-\left(\mathbf{T}^{-1}+\Phi\right)^{-1}\left(\mathbf{T}^{-1}+\Phi^{*}\right)
$$

The following lemma describes the set of all selfadjoint extensions of the operator $A_{M}^{0}$ which can be obtained as finite rank additive perturbations of the operator $A$ providing that the family of singular vectors $\varphi_{j}$ from $\mathcal{H}_{-1}(|A|)$ remains unchanged.

Lemma 2. Let $\varphi_{j} \in \mathcal{H}_{-1}(|A|) \backslash \mathcal{H}, j=1,2, \ldots, M$, form an orthonormal system in $\mathcal{H}_{-2}(A)$. If $\operatorname{det}\left(V-\frac{i-\Re \Phi}{i+\Re \Phi}\right) \neq 0$, then the operator $A_{M}^{0 *}$ restricted to the domain of functions $\left\{\psi \in \operatorname{Dom}\left(A_{M}^{0 *}: \vec{a}_{-}(\psi)=V \vec{a}_{+}(\psi)\right\}\right.$ is a finite dimensional additive perturbation of the operator $A$.

Proof. Suppose that the matrix $I+V$ is invertible $(\operatorname{det}(I+V) \neq 0)$. Then the matrix $\mathbf{T}^{-1}$ can be calculated as follows using the representation (15):

$$
\mathbf{T}^{-1}=-\Phi\left(I+V^{*}\right)^{-1}-\Phi^{*}(I+V)^{-1}=-\Re \Phi+i \frac{I-V}{I+V} .
$$

The determinant of the matrix in the right hand side of the latter equality is not equal to zero:

$$
\operatorname{det}\left(-\Re \Phi+i \frac{I-V}{I+V}\right)=-(\operatorname{det}(I+V))^{-1} \operatorname{det}(\Re \Phi+i) \operatorname{det}\left(V-\frac{i-\Re \Phi}{i+\Re \Phi}\right) .
$$

Then the Hermitian matrix $-\Re \Phi+i \frac{I-V}{I+V}$ is invertible, and the matrix $\mathbf{T}$ can be reconstructed as follows:

$$
\mathbf{T}=\left(-\Re \Phi+i \frac{I-V}{I+V}\right)^{-1}
$$

$\mathbf{T}$ is then Hermitian.

Consider now the case where $\operatorname{det}(I+V)=0$. This equality implies that the matrix $V$ has a nontrivial eigensubspace $N_{-1}$ corresponding to the eigenvalue -1 . The restriction of the adjoint operator $A_{M}^{0 *}$ to the subspace

$$
\left\{\psi \in \operatorname{Dom}\left(A_{M}^{0 *}\right): P_{N_{-1}} \vec{a}_{+}(\psi)=-P_{N_{-1}} \vec{a}_{-}(\psi)\right\}
$$

coincides with the restriction of the operator $A$ to the same subspace. Thus the set of vectors $\left\{\varphi_{j}\right\}_{j=1}^{M}$ contains extra elements in the following sense: one can find some new set of elements $\left\{\varphi_{j}^{\prime}\right\}_{j=1}^{M^{\prime}}, M^{\prime}<M$, such that the corresponding matrix $V^{\prime}$ has a trivial eigensubspace $N_{-1}^{\prime}$. We have already proven that every unitary matrix which does not have eigenvalue -1 describes a certain finite rank perturbation of the original operator. The lemma is proven.

This last lemma characterizes the set of selfadjoint extensions of the operator $A_{M}^{0}$ which are finite rank perturbations of the original operator. The selfadjoint extensions corresponding to the matrices $V$ satisfying the equality $\operatorname{det}\left(V-\frac{i-\Re \Phi}{i+\Re \Phi}\right)=0$ can be described by finite rank perturbations with infinite strength using the projective space formalism. This approach is developed in [15], where point interactions for the second derivative operator in one dimension are studied. 


\section{FORM UNBOUNDED FINITE RANK PERTURBATIONS}

Form unbounded finite rank perturbations can be described following the main ideas developed in $[5,6]$ for rank one perturbations. We suppose in this section that the vectors $\varphi_{j}$ in the representation (5) are elements from the Hilbert space $\mathcal{H}_{-2}(A)$ and that the matrix $\mathbf{T}$ is Hermitian and invertible. The quadratic form defined by the perturbation is not necessarily bounded with respect to the quadratic form of the operator $A$. The unique selfadjoint extension of the operator $A_{M}^{0}$ corresponding to the expression (5) defined on the domain $\operatorname{Dom}(A)$ can be determined only if all elements $\varphi_{j}$ can be extended as bounded linear functionals to the domain $\mathrm{B}_{M}(A)=\operatorname{Dom}\left(A_{M}^{0 *}\right)$. The space $\mathrm{B}_{M}(A)$ is a finite dimensional extension of the space $\mathcal{H}_{-2}(A)=\operatorname{Dom}(A)$. Thus the extensions are defined if the following coefficients are determined:

$$
\Phi_{i j}=\left\langle\varphi_{i}, \frac{1}{A-i} \varphi_{j}\right\rangle=\left\langle\varphi_{i}, \frac{A}{A^{2}+1} \varphi_{j}\right\rangle+i\left\langle\varphi_{i}, \frac{1}{A^{2}+1} \varphi_{j}\right\rangle .
$$

The second scalar product on the right hand side of the latter equality is well defined, since $\varphi_{j}, \varphi_{i} \in \mathcal{H}_{-2}(A)$. Therefore to determine the matrix $\Phi$ it is enough to find a Hermitian matrix $\mathrm{R}$ with the coefficients

$$
\mathrm{R}_{i j}=\left\langle\varphi_{i}, \frac{A}{A^{2}+1} \varphi_{j}\right\rangle
$$

provided these are well defined.

Let us denote by $\mu_{\varphi_{i}, \varphi_{j}}(\lambda)$ the spectral measure corresponding to the elements $\varphi_{i}, \varphi_{j}$ and the operator $A$ :

$$
\begin{aligned}
\left\langle\frac{1}{A-z_{i}} \varphi_{i}, \frac{1}{A-z_{j}} \varphi_{j}\right\rangle & =\int_{-\infty}^{\infty} \frac{1}{\lambda-\bar{z}_{i}} \frac{1}{\lambda-z_{j}} d \mu_{\varphi_{i}, \varphi_{j}}(\lambda) \\
\int_{-\infty}^{\infty} \frac{1}{\lambda^{2}+1} d \mu_{\varphi_{i}, \varphi_{j}}(\lambda) & \leq\left\|\varphi_{i}\right\|_{\mathcal{H}_{-2}(A)}\left\|\varphi_{j}\right\|_{\mathcal{H}_{-2}(A)}<\infty .
\end{aligned}
$$

If the integral $\int_{-\infty}^{\infty} \frac{\lambda}{\lambda^{2}+1} d \mu_{\varphi_{i}, \varphi_{j}}(\lambda)$ is absolutely convergent, then the scalar product $\left\langle\varphi_{i}, \frac{A}{A^{2}+1} \varphi_{j}\right\rangle$ is indeed well defined. The latter integral converges absolutely if both vectors $\varphi_{i}, \varphi_{j}$ are elements from the space $\mathcal{H}_{-1}(|A|)$. The same is true if the following inclusions hold: $\varphi_{i} \in \mathcal{H}_{-2,0}(A), \varphi_{j} \in \mathcal{H}_{0,-2}(A)$. (The spaces $\mathcal{H}_{n, m}(A)$ denote the vector spaces of elements having spectral projections to the negative, resp. positive, halfaxis belonging to $\mathcal{H}_{n}(|A|)$, resp. $\mathcal{H}_{m}(|A|)$. )

Definition 1. The Hermitian matrix $\mathrm{R}$ corresponding to the set of vectors $\varphi_{j} \in$ $\mathcal{H}_{-2}(A), j=1, \ldots, M$, is called admissible if the equality

$$
\left\langle f, \frac{A}{A^{2}+1} g\right\rangle=\sum_{i, j=1}^{M} \bar{f}_{j} \mathrm{R}_{j i} g_{i}
$$

holds for every two functions $f$ and $g$ provided that

- $f$ and $g$ are elements from the linear hull of the vectors $\varphi_{j}$, i.e.

$$
f=\sum_{j=1}^{M} f_{j} \varphi_{j}, \quad g=\sum_{j=1}^{M} g_{j} \varphi_{j}
$$


- the spectral measure $\mu_{f, g}$ corresponding to the functions $f$ and $g$ determines the absolutely convergent integral

$$
\int_{-\infty}^{\infty} \frac{\lambda}{\lambda^{2}+1} d \mu_{f, g}(\lambda)<\infty
$$

There exist sets of vectors $\varphi_{j}$ such that for no two linear combinations of them does the integral (23) converge. Every Hermitian matrix $\mathrm{R}$ is admissible in this case. If all the vectors $\varphi_{j}$ are elements from the Hilbert space $\mathcal{H}_{-1}(|A|)$ then the admissible matrix $\mathrm{R}$ is unique, as we have already seen in the previous section.

We shall consider in what follows only admissible Hermitian matrices R. The following theorem can be proven

Theorem 2. Let $\varphi_{j} \in \mathcal{H}_{-2}(A) \backslash \mathcal{H}$ and let $\mathbf{T}=\left\{t_{i j}\right\}_{i, j=1}^{M}$ be a Hermitian invertible matrix. Suppose that the Hermitian matrix $\mathrm{R}=\left\{\mathrm{R}_{i j}\right\}_{i, j=1}^{M}$ is admissible for the set of vectors $\varphi_{j}, j=1, \ldots, M$. Then the selfadjoint operator $A_{T}=$ $A+\sum_{i, j=1}^{M} t_{i j}\left\langle\varphi_{j}, \cdot\right\rangle \varphi_{i}$ is the selfadjoint extension of the operator $A_{M}^{0}$ to the following domain:

$$
\operatorname{Dom}\left(A_{T}\right)=\left\{\psi \in \operatorname{Dom}\left(A_{M}^{0 *}\right): \vec{a}_{+}(\psi)=-\left(\mathbf{T}^{-1}+\Phi\right)^{-1}\left(\mathbf{T}^{-1}+\Phi^{*}\right) \vec{a}_{-}(\psi)\right\},
$$

where $\Phi=\mathrm{R}+i I$.

Proof. The proof is very similar to that of Theorem 1.

We note that the domain of the operator $A_{T}$ depends on the choice of the admissible matrix $\mathrm{R}$. If all the vectors $\varphi_{j}$ are elements from the Hilbert space $\mathcal{H}_{-1}(|A|)$, then the admissible matrix $\mathrm{R}$ is unique and there is a unique selfadjoint operator corresponding to the formal expression (5). This case has been studied in section 3 .

The unique admissible matrix $\mathrm{R}$ can be determined by some extra conditions even if the vectors $\varphi_{j}$ are not elements from the Hilbert space $\mathcal{H}_{-1}(|A|)$. For example, such a unique matrix exists if all the vectors $\varphi_{j}$ and the operator $A$ are homogeneous with respect to a certain group of unitary transformations. Consider for example the operator in $L_{2}(\mathbf{R})$ formally given by $\left(i \frac{d}{d x}\right)^{n}+\sum_{k=0}^{n-1} \alpha_{k} \delta^{(k)}$, where $\delta^{(k)}$ are the derivatives of the delta function. Not all $\delta^{(k)}, k=1,2, \ldots, n-1$, are from the Hilbert space $\mathcal{H}_{-1}\left(\left|\left(i \frac{d}{d x}\right)^{n}\right|\right)$. Therefore the operator corresponding to the formal expression can be uniquely defined using only the fact that the original operator and the distributions $\delta^{(k)}$ are homogeneous with respect to the scaling group. This approach has been developed for second order differential operators in [15] and for operators of arbitrary order in [8].

The approach developed in this paper can be used to construct model few-body operators with $\mathcal{H}_{-1}$ and $\mathcal{H}_{-2}$ cluster interactions. Such operators can be obtained as infinite dimensional perturbations of the operators describing the system of noninteracting particles ([7]).

\section{ACKNOWLEDGMENTS}

The financial support of the Alexander von Humboldt Foundation for the second author is gratefully acknowledged. 


\section{REFERENCES}

[1] N.I.Akhiezer, I.M.Glazman, "Theory of linear operators in Hilbert Space", vol I, II, Pitman, London, 1981. MR 83i:47001

[2] S.Albeverio, F.Gesztesy, R.Høegh-Krohn, H.Holden, "Solvable models in quantum mechanics", Springer-Verlag, 1988. MR 90a:81021

[3] S.Albeverio, W.Karwowski, V.Koshmanenko, Square powers of singularly perturbed operators, Math. Nachr. 173, 5-24 (1995). MR 97a:47035

[4] S.Albeverio, V.Koshmanenko, Some remarks on the Gesztesy-Simon version of rank one perturbations, Kiev Preprint (1996).

[5] S.Albeverio, P.Kurasov, Rank one perturbations, approximations and selfadjoint extensions, J. Func. Anal. 148, 152-169 (1997). MR 98g:47011

[6] S.Albeverio, P.Kurasov, Rank one perturbations of not semibounded operators, Integr. Eq. Oper. Theory 27, 379-400 (1997). CMP 97:10

[7] S.Albeverio, P.Kurasov, "Solvable Schrödinger type operators. Singular perturbations of differential operators", Cambridge Univ. Press, to appear.

[8] J.Boman, P.Kurasov, Finite Rank Singular Perturbations and Distributions with Discontinuous Testfunctions, Proceedings AMS 126 (1998), 1673-1683. MR 98g:47012

[9] F.Gesztesy and B.Simon, Rank one perturbations at infinite coupling, J. Func. Anal 128, 245-252 (1995). MR 95m:47014

[10] S.Hassi, H.Langer, H. de Snoo, Selfadjoint extensions for a class of symmetric operators with defect numbers $(1,1)$, in: A.Gheondea, S.Stratila, D. Timotin (eds.), "Topics in Operator Theory, Operator Algebras and Applications", Inst. of Mathematics of the Romanian Academy, Bucharest, 1995, pp. 115-145. MR 97m:47024

[11] S.Hassi, H. de Snoo, On rank one perturbations of selfadjoint operators, Integr. Eq. Oper. Theory 29, 288-300 (1997). CMP 98:03

[12] L.Hörmander, "The Analysis of Linear Partial Differential Operators I", Springer-Verlag, Berlin, 1983. MR 85g:35002a

[13] A.Kiselev, and B.Simon, Rank one perturbations with infinitesimal coupling, J. Func. Anal 130, 345- 356 (1995). MR 96e: 47012

[14] V.D.Koshmanenko, Toward the Rank-one Singular Perturbations Theory of Self-adjoint Operators, Ukranian Math. J. 43, No 11, 1450-1457 (1991). MR 93b:47020

[15] P.Kurasov, Distribution theory for the discontinuous test functions and differential operators with the generalized coefficients, J.Math. Anal. Appl. 201, 297-323 (1996) . MR 97g:46050

[16] P.Kurasov, Rank one perturbations of nonsemibounded operators, Research report 1995-15, Luleå University of Technology, Luleå, Sweden, 1995.

[17] M.G.Krein, Concerning the resolvents of an Hermitian operator with the deficiency-index $(m, m)$, C. R. (Dokl.) Akad. Sci. URSS 52, 651-654 (1946). MR 8:277a

[18] M.Reed, B.Simon, "Methods of modern mathematical physics", vol. II, Academic Press, New York, 1975. MR 58:12429b

[19] B.Simon, Spectral Analysis of Rank One Perturbations and Applications, CRM Proceedings and Lecture Notes, Volume 8, 109-149 (1995). MR 97c:47008

Department of Mathematics, Ruhr-University Bochum, 44780 Bochum, Germany; SFB 237 Essen-Bochum-Düsseldorf, Germany; BiBoS Research Center, D 33615 Bielefeld, Germany; CERFIM, Locarno, Switzerland

Current address: Institute of Applied Mathematics, University of Bonn, Bonn, Germany

E-mail address: albeverio@uni-bonn.de

Department of Mathematics, Stockholm University, 10691 Stockholm, Sweden; Alexander von Humboldt fellow, Department of Mathematics, Ruhr-University Bochum, 44780 Bochum, Germany; Department of Mathematical and Computational Physics, St.Petersburg University, 198904 St.Petersburg, Russia; Department of Mathematics, Lule̊ University, 97187 Lule̊̊, Sweden 